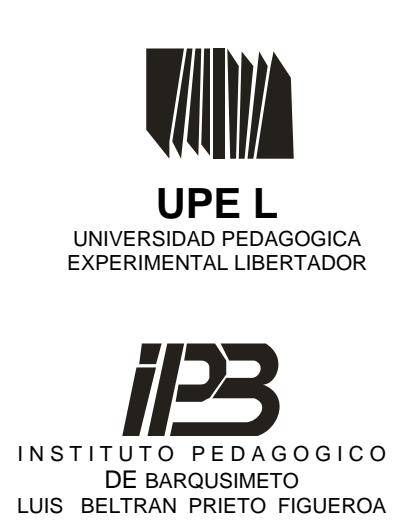

NUEVA ETAPA

FORMATO ELECTRÒNICO

DEPOSITO LEGAL: ppi201002LA3674

ISSN: 2244-7296

\author{
REVISTA

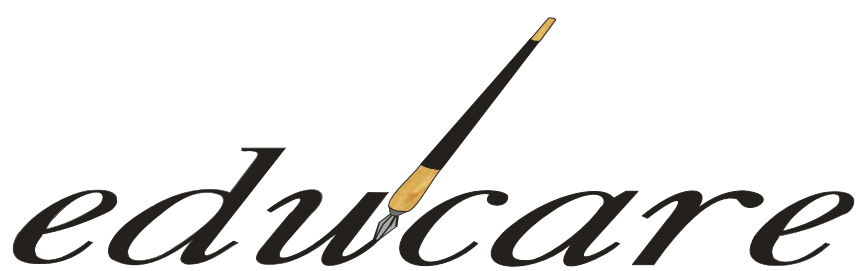 \\ Órgano Divulgativo de la Subdirección de Investigación y Postgrado \\ del Instituto Pedagógico de Barquisimeto "Luis Beltrán Prieto \\ Figueroa"
}

\title{
EL PORTAFOLIO COMO ALTERNATIVA DE EVALUACIÓN EN INGLÉS PARA PROPÓSITOS ESPECÍFICOS
}

Portfolio as assessment alternative in English for Specific Purposes

\author{
Bexi Perdomo
}

Universidad de Los Andes ( ULA) 


\title{
EL PORTAFOLIO COMO ALTERNATIVA DE EVALUACIÓN EN INGLÉS PARA PROPÓSITOS ESPECÍFICOS*
}

\author{
PORTFOLIO AS ASSESSMENT ALTERNATIVE IN ENGLISH FOR \\ SPECIFIC PURPOSES
}

\begin{abstract}
Bexi Perdomo**
ULA

INVESTIGACIÓN
\end{abstract}

Recibido : 16-09-2010

Aceptado: $12-12-2010$

\section{RESUMEN}

El portafolio ha sido recomendado para la evaluación en la enseñanza del inglés como lengua extranjera, pero pocas investigaciones se han publicado sobre su efectividad en dicho contexto y compararlo con otras estrategias de evaluación comúnmente utilizadas en el aula. El objetivo de esta investigación, fue evaluar el uso del portafolio y actividades prácticas en el aula como estrategias de evaluación en cursos de inglés para propósitos específicos. En este estudio cuasiexperimental y transversal participaron 196 estudiantes cursantes del primer año de su carrera universitaria. Los resultados indican que el portafolio es una alternativa que favorece el rendimiento de los estudiantes en comparación con trabajos prácticos hechos y entregados en el aula. Se sugiere usar el portafolio haciendo énfasis en la motivación al estudiante acerca de las bondades de esta estrategia de evaluación y resaltar la necesidad de seguir adecuadamente las instrucciones para incrementar el éxito de la estrategia.

Descriptores: Inglés para propósitos específicos (IPE), Inglés como lengua extranjera (ILE), portafolio, evaluación, constructivismo.

\begin{abstract}
Portfolio has been recommended for the evaluation and assessment in the teaching of English as a foreign language, but few studies have been published about its effectiveness of portfolio in the aforementioned context and to compare it to other evaluation strategies commonly used in the classroom. This study aimed to compare the use of portfolio and practice activities delivered in class for the evaluation of two ESP courses. Participants in this cuasiexperimental and cross-sectional research were 196 university students enrolled in the first year. Results show that portfolio is a choice that enhances students' performance when compared to tasks done and delivered in class. It is suggested to use portfolio making emphasis in motivating students about the benefits of this evaluation strategy. It is also necessary to highlight the importance of following instructions to be more successful when using this strategy.
\end{abstract}

Key words: English for Specific Purposes (ESP), English as a Foreign Language (EFL), portfolio, evaluation, constructivism.

\footnotetext{
* Parte de esta investigación fue presentada en el ICERI 2008 en Madrid, España (Con el apoyo financiero del CDCHT de la ULA, Venezuela).

** Prof. Agregado en el Dpto. De Investigación de la Facultad de Odontología de la Universidad de Los Andes, Venezuela. Miembro del Grupo Multidisciplinario de Investigaciones en Odontología (G-MIO). Correo electrónico: bexi@ula.ve
} 


\section{INTRODUCCIÓN}

Como consecuencia de los cambios curriculares y metodológicos que han tenido lugar en los últimos años en Venezuela, las actividades académicas han venido desarrollándose cada vez más ambientes constructivistas en los que, al igual que en otros contextos, se requiere congruencia tanto entre las actividades de clase, complementarias y de evaluación, y las premisas que sustentan dicho entorno de aprendizaje. Esta concepción del conocimiento como objeto de construcción y no de trasmisión, ha repercutido en los ámbitos teóricos y metodológicos y ha generado cambios notables en las aproximaciones a la enseñanza de las lenguas extranjeras (Delmastro y Salazar, 2004). Esto hace necesario que tanto las estrategias didácticas como los materiales, instrumentos y técnicas de evaluación sean pertinentes para este ambiente de aprendizaje.

Dentro de un enfoque constructivista los alumnos aprenden haciendo y experimentando y la evaluación en este ambiente consiste en obtener información acerca de los descubrimientos y avances del estudiante y su grado de apropiación de la estructura básica de la ciencia al final del proceso (Floréz, 1999). Así, actividades como trabajos en pareja o grupos en el aula, elaboración de portafolios, exposiciones, investigaciones, entre otras, se adecuan para un curso en el cual se persigue que el estudiante aprenda haciendo y alcance aprendizajes significativos.

Como puede observarse, entre las estrategias de evaluación apropiadas para este entorno se encuentra los trabajos en equipo y el portafolio. Éste último según algunos autores fomenta la autonomía del estudiante en el proceso de aprendizaje (Barragán, 2005; Lunar, 2007; Öztürk y Çeçen, 2007 y Autor, 2008b) y le permite generar criterios acerca de la prioridad de las actividades en función de sus necesidades de aprendizaje (Barragán, 2005).

El portafolio ha sido considerado en la literatura como una herramienta útil tanto para la enseñanza como para la evaluación en cualquier asignatura (Villalobos, 2002 y Lau, 2006). Diferentes autores, (por ejemplo Delmastro, 2005a, 2005b y Autor, 2008b) han hablado en favor del uso del portafolio en el contexto de la enseñanza de Inglés para Propósitos Específicos (IPE) y su efectividad para la evaluación en el mismo contexto. Por su parte, Barragán (2005) señala que el portafolio es un instrumento que hace posible evaluar procesos 
y productos, motivar los estudiantes a pensar sobre su propio proceso de aprendizaje al participar en el proceso de evaluación, desarrollar destrezas colaborativas entre estudiantes, promover habilidades para la resolución de problemas, entre otros. Sin embargo, Barragán no indica si el beneficio del uso de portafolio incluye ayudar a los estudiantes a obtener mejores calificaciones en comparación a otros tipos de evaluaciones. Adicionalmente, Kavaliauskiene, Kaminskiene y Anusiene (2007) afirman que el portafolio contribuye en la formación de estudiantes cada vez más autónomos, acordes a los enfoques de enseñanza actuales, incluidos los eclécticos que involucran el constructivismo (Delmastro y Salazar, 2004).

El proceso de enseñanza de inglés como Lengua Extranjera (ILE) y específicamente Inglés para Propósitos Específicos (IPE) puede darse dentro de un ambiente basado en premisas constructivistas. Cuando este es el caso, los procesos de evaluación, deben incluir estrategias e instrumentos consistentes con la concepción del aprendizaje sobre las cuales se fundamenta la práctica docente. En este sentido, Reeves y Okey (1996) señalan que en los contextos constructivistas en necesario ampliar las alternativas para la evaluación de los aprendizajes, tanto desde el punto de vista de proceso como de producto y en este contexto el portafolio se perfila como una alternativa plenamente ajustable. Sin embargo, pese a la relevancia del tema y sus implicaciones pedagógicas, en Venezuela existen pocas investigaciones de campo relacionadas con el uso de ciertas estrategias para la enseñanza de IPE (según búsqueda en los repositorios de las diferentes universidades venezolanas y diferentes revistas nacionales impresas), por lo que aún es necesario investigar acerca de la efectividad y adecuación de diferentes estrategias de evaluación en el contexto antes mencionado.

Del portafolio se ha escrito acerca de la dificultad de incorporar el mismo en el aula de IPE, ya que su implementación demanda mucho tiempo y esfuerzo por parte del profesor (Song y August, 2002; Lau, 2006; Vera y Canalejas, 2007 y Autor, 2008b; entre otros). No obstante, en recientes investigaciones en Venezuela, los estudiantes universitarios han manifestado sentirse cómodos siendo evaluados con esta estrategia (Autor, 2008b) lo cual habla en favor de su uso y es consistente con lo presentado por otros autores en diferentes contextos (Barragán, 2005 y Lau, 2006). En medio de este conjunto de conclusiones a las que se ha llegado en estudios previos, se percibe la necesidad de evaluar diferentes aspectos del 
portafolio a fin de poder decidir si los beneficios que éste aporta a los estudiantes justifican la inversión de esfuerzo y tiempo que toman del docente o si por el contrario al funcionar igual o peor que otras estrategias es preferible sustituirlo por éstas.

En el contexto venezolano, el portafolio ha sido propuesto en la última década como una herramienta de evaluación por parte de algunos docentes e investigadores venezolanos (por ejemplo, Delmastro 2005a y 2005b, Lunar, 2007 y Autor 2008b). Sin embargo, en Venezuela se han publicado pocos artículos relacionados con su uso para la evaluación en el contexto de IPE, pese a la necesidad de investigar sobre las estrategias de evaluación idóneas para favorecer el aprendizaje en un contexto constructivista. En consecuencia, se requiere realizar investigaciones con el propósito de estudiar el portafolio en comparación con otras estrategias de evaluación también ajustables a contextos caracterizados por premisas constructivistas y frecuentemente usados en las aulas de clase. De esta forma, el docente se permite incorporar estrategias e instrumentos de evaluación que puedan motivar a los estudiantes, que sean acordes para el contexto educativo y se ajusten las necesidades de los educandos.

En tal sentido, es importante que el docente se convierta en un ensayador de propuestas de enseñanza y de estrategias para comprobar en la práctica si funcionan o no, a fin de obtener y ponderar del conjunto de cada experiencia pedagógica una información de retorno para el grupo actual y para los grupos que se le presenten en el futuro (Floréz, 1999). Dicho en otras palabras, el profesor debe acompañar el acto docente con el acto investigativo tanto de la literatura existente como sobre la base de su propia práctica en el aula. Es así como surgió esta investigación cuyo objetivo fue evaluar el uso de dos estrategias de evaluación (portafolio y actividades prácticas en el aula) en dos cursos de IPE, partiendo de la hipótesis de que el portafolio tendría una repercusión positiva sobre el rendimiento académico de los estudiantes, la cual se vería reflejada en sus calificaciones. 


\section{ARGUMENTACION TEORICA}

\section{Estudios previos}

En Venezuela son pocos los investigadores que se han dedicado al estudio del portafolio en el contexto de ILE, pese a que se ha argumentado en favor de su incorporación en el aula, esta afirmación se fundamenta en una búsqueda en las revistas y bases de datos de revistas científicas venezolanas en un periodo de 10 años (2000-2010). De esta manera se aprecia que no se cuenta con muchos estudios previos acerca del tema por lo que lo que a continuación se presenta constituye una breve revisión general dentro y fuera del contexto venezolano.

Entre los investigadores pioneros en los estudios del portafolio en la enseñanza de lenguas extranjeras en Venezuela se encuentra Akirov (1997) quien publicó un material en el cual discutía las ventajas del portafolio en el contexto de ILE, particularmente en lo atinente a la enseñanza de la escritura y la lectura. Posteriormente, se detuvo la publicación sobre el tema y años más tarde, Delmastro (2005ª) presentó un artículo en el cual describió cómo percibían los docentes el uso del portafolio para trabajo y evaluación y sugirió orientaciones para su uso en el aula de lenguas extranjeras. Los resultados indicaron que los docentes consideran que los estudiantes asumen una actitud pasiva y carente de compromiso, lo cual afecta significativamente el uso exitoso de esta estrategia.

Como parte del mismo proyecto de investigación, Delmastro (2005b) publicó otro estudio en el cual se había planteado tres objetivos: identificar los fundamentos teóricos que sustentan el uso del portafolio en el desarrollo y evaluación de destrezas en lengua extranjera, analizar las ventajas y los inconvenientes potenciales señalados en la literatura en situaciones de enseñanza de lengua extranjera y finalmente, identificar lineamientos procedimentales que orienten su aplicación en cursos de lenguas extranjeras. La investigación mostró que el uso del portafolio se fundamenta en los principios del constructivismo y enfoques contemporáneos para la enseñanza de lengua extranjera. En esta oportunidad, la autora concluyó que era necesario promover entre los docentes el uso de esta estrategia.

Por otra parte, en el contexto extranjero, Makarchuck (2003) examinó el valor de portafolio en un curso conversacional de inglés en una universidad pública en Corea del Sur 
en relación con su impacto sobre el aprendizaje desde la perspectiva de los estudiantes y como estrategia de evaluación desde el punto de vista del facilitador del curso. Este estudio se llevó a cabo con 39 estudiantes universitarios y en el mismo se le permitió a los participantes comparar ambas condiciones (sin portafolio y con portafolio en la primera y segunda mitad del semestre respectivamente). Al igual que en la presente investigación, el facilitador dio instrucciones claras acerca de la forma de elaborar y presentar el portafolio y resaltó la importancia de hacerlo de acuerdo a las instrucciones dadas. Los resultados indicaron que los estudiantes consideran los portafolios como un elemento positivo a agregar en el aula y apenas un 15\% indicó no haberlo disfrutado. Adicionalmente, en términos de evaluación, el portafolio mostró tanto fortalezas como limitaciones que son importantes de considerar al momento de decidir su inclusión en el aula. Makarchuck (2003) no encontró diferencias significativas entre la evaluación con estrategias tradicionales ajustables al constructivismo y la evaluación que incluía portafolios.

También Peña, Ball y Delhi (2005) abordaron el tema, pero desde una perspectiva documental. Su objetivo fue discutir aspectos teóricos fundamentales en el uso del portafolio. En su publicación incluyeron orientaciones para la elaboración por parte del profesor y del estudiante. Los autores señalaron aspectos que podrían incluirse en ambos portafolios, y argumentan que los mismos elementos pueden variar dependiendo de la naturaleza de la asignatura y del propósito para el cual se elabora. Concluyeron que para que el portafolio cumpla con su finalidad se debe tomar en cuenta las dimensiones teóricas y prácticas del quehacer pedagógico.

Más recientemente, en Barcelona España, Vera y Canalejas (2007) realizaron una investigación cuyo objetivo fue estudiar el uso del portafolio como un recurso para impulsar la autodirección de los estudiantes en el proceso de aprender a aprender y potenciar la construcción personal del conocimiento. Los resultados de este estudio demostraron que todos los estudiantes, a excepción de uno, progresaron en la construcción de conocimiento y en la capacidad de autodirigir su aprendizaje. Los autores concluyeron que el portafolio ayuda, entre otras cosas, a adquirir competencias tales como aprendizaje autodirigido. Dada la dificultad que representa para un solo docente corregir tantos portafolios, los autores recomiendan que en aquellos cursos donde los grupos sean muy grandes se elaboren los 
portafolios por pequeños equipos. Aún cuando el estudio de Vera y Canalejas (2007) no fue en el área de IPE sino en una asignatura de la carrera de enfermería es importante considerarlo dados sus resultados como herramienta promotora del aprendizaje autodirigido y las recomendaciones prácticas para los docentes.

Las investigaciones acerca del portafolio en la enseñanza de ILE, han ido diversificándose en el contexto internacional y han ido más allá de la caracterización de su uso. Es así como Öztürk y Çeçen (2007) investigaron el efecto del portafolio sobre la ansiedad en estudiantes para profesores de inglés como lengua extranjera en Turquía y usaron la escala para medir la ansiedad en escritura (SLWAI). Estos autores encontraron que el hecho de construir un portafolio fue beneficioso para los estudiantes en cuanto a superar su ansiedad en la escritura. De igual manera observaron que en términos generales, el portafolio influye en los estudiantes de forma positiva.

Como puede observarse al revisar la literatura, en líneas generales sigue habiendo vacíos en cuanto a evidencia empírica que dé cuenta de la efectividad del portafolio en los contextos de IPE. Particularmente en Venezuela, se ha publicado muy poco acerca de esta estrategia alternativa de evaluación que es congruente con ambientes constructivistas de enseñanza y aprendizaje en las aulas de IPE.

\section{Algunas consideraciones teóricas sobre los portafolios}

Por definición, los portafolios son una representación de la práctica constructivista (Öztürk y Çeçen, 2007). Delmastro (2005 ) señala que el portafolio viene a ser un soporte a los enfoques intruccionales basados en premisas constructivistas que hacen énfasis en el rol activo del estudiante en la construcción de conocimientos y del profesor como un mediador en ese proceso de construcción. Algunos autores conciben los portafolios, tanto escritos como digitales como parte de la lista de evaluaciones alternativas para contextos eclécticos o constructivistas (Kavaliauskiene et al., 2007).

El portafolio ha sido considerado tanto como herramienta para la enseñanza como para la evaluación. En consecuencia, la investigación acerca del uso del portafolio en la enseñanza y el aprendizaje de EFL se ha conducido desde diferentes perspectivas. Entre las bondades del 
portafolio señaladas en la literatura, se encuentra que puede generar la reflexión en los estudiantes y ayudar significativamente en el propio monitoreo de su aprendizaje, contribuyendo a que sean autónomos en su proceso de aprendizaje (Nunes, 2004). Lau (2006) agrega que el portafolio como herramienta de evaluación centrada en los estudiantes da a éstos la oportunidad de construir sus experiencias en el proceso de aprendizaje y que esta experiencia puede motivarles a sentirse más involucrados en dicho proceso puesto que sienten que tienen control sobre su propio proceso de aprendizaje.

En cuanto a lo que se incluirá en los portafolios, Barragán (2005) y Autor (2008b) indican que no hay lineamientos fijos y que estos pueden ser establecidos tanto por los alumnos como por los docentes o producto de un acuerdo entre ambos. Adicionalmente, en la literatura se ha hablado acerca de la importancia de que los estudiantes estén claros en el propósito del portafolio y de darle instrucciones claras acerca de la forma de elaborarlo y presentarlo (Villalobos, 2002). Por lo tanto, se ha hecho énfasis de que dichas instrucciones sean tanto verbales como escritas y disponibles tanto en formato impreso como electrónico (Autor, 2008b). Además, es necesario que el docente comunique claramente tanto los contenidos a trabajar en el portafolio como la forma de hacerlo y debe asegurarse de que se haya comprendido el mensaje para evitar confusión y frustración en los estudiantes (Lau, 2006).

Ahora bien, en lo atinente a los beneficios que el portafolio ofrece en el aula de inglés como lengua extranjera, Barragán (2005) enumera algunos:

a) Permite evaluar tanto proceso como producto.

b) Motiva a los estudiantes a reflexionar sobre su propio proceso de aprendizaje al involucrarlos en su propia evaluación con mayor autonomía.

c) Permite desarrollar destrezas colaborativas entre los estudiantes.

d) Facilita la identificación adecuada de cada tarea a realizar, identificando con claridad lo que es indispensable y lo que no.

e) Da a los profesores la oportunidad de adecuar los contenidos y estrategias de trabajo a las necesidades reales de los estudiantes.

A estos beneficios, según Barragán (2005) y Autor (2008b) se puede adicionar que el portafolio es una forma de evaluación con mayor aceptación que los exámenes tradicionales 
en los estudiantes. Adicionalmente, el portafolio como un medio para la evaluación ha ido ganando el interés de los profesores que desean incluir formas alternativas de evaluar el conocimiento y las destrezas de sus estudiantes, para hacer que ellos participen activamente en el proceso de evaluación y simultáneamente desarrollar destreza de pensamiento reflexivo (Kavaliauskiene et al., 2007).

Por otra parte, en la literatura también se ha hablado acerca de las debilidades del portafolio para fines de evaluación. Al respecto, Kavaliauskiene et al. (2007) hacen referencia a su baja comparabilidad y confiabilidad, así como la dificultad de garantizar condiciones de evaluación estandarizadas. Otra debilidad del uso del portafolio es la cantidad de esfuerzo, tiempo y trabajo que requiere de los docentes (Autor, 2008b; Vera y Canalejas, 2007; entre otros).

Finalmente, otro aspecto importante de conocer acerca de los portafolios es que estos pueden ser de varios tipos dependiendo del propósito para el cual son solicitados, el material que incluyen y el momento en que se evalúan. Entre esta última clasificación se encuentran los portafolios de trabajo y de producto. El primero se refiere a aquél en el que se incluyen todos los borradores de sus trabajos y son básicamente usados para evaluar procesos; mientras que el segundo hace referencia a un portafolio en el que sólo se exhibe el producto terminado o la mejor versión alcanzada por el alumno. Aun cuando el segundo permite al docente evaluar sólo la versión final, sigue dando la oportunidad al estudiante de apreciar su evolución y de autoevaluarse hasta decidir cuál versión incluir. Otra clasificación está en función del enfoque con el que se decide evaluar; desde esta perspectiva el portafolio puede tener fines de evaluación formativa o sumativa. En este sentido, el docente debe documentarse acerca de los tipos de portafolios y decidir con cuál trabajará en función de los objetivos en torno a los cuales plantea su uso.

\section{ASPECTOS METODOLÓGICOS}

\section{Diseño del estudio}

Se realizó un estudio cuasiexperimental, de acuerdo a la clasificación de diseños de investigación de León y Montero (1997) en el ámbito educativo. En este sentido, cada uno de los sujetos no pudo ser asignado al azar a las condiciones sino que se trabajó con lo que se 
conoce en investigación de este tipo como grupos intactos, los cuales fueron designados al azar a una de las dos condiciones de experimentación (trabajo en aula o portafolio). La investigación fue transversal, ya que en cada grupo se hizo una sola medición y no se analizó la evolución en el tiempo para cada uno.

\section{Participantes}

Colaboraron en esta investigación ciento noventa y seis estudiantes universitarios en su primer año de la carrera divididos en dos grupos ( $\mathrm{n}=98$ cada uno). Los mismos pertenecían a diferentes cohortes pero compartían características similares desde el punto de vista social, económico, educativo y del dominio del idioma para el momento en que se inició el curso y se hizo el estudio. Estos estudiantes participaron en una de las dos condiciones de evaluación: 1) portafolio y 2) actividades prácticas a realizar y entregar en clase.

De esta forma, se consideraron en el estudio los estudiantes que se tuvieran características etáreas, socioculturales y antecedentes educativos similares, para evitar posibles efectos de variables intervinientes. No se tuvieron que excluir datos de ningún sujeto, ya que como observó Autor (2007) en un estudio previo, los estudiantes de nuevo ingreso de la FOULA guardan características similares (en cuanto a los rasgos señalados) de cohorte en cohorte.

\section{Instrumentos de recolección de datos}

Durante la primera semana de clase, los estudiantes llenaron una ficha que incluía información personal. En ésta sólo se preguntó acerca de sus antecedentes económicos, sociales y educativos con la finalidad de asegurarse de que no participaran sujetos con diferencia marcadas con respecto al resto del grupo en algunos de estos aspectos. Esta ficha fue previamente sometida a una validación de contenido a través de la técnica de juicio de expertos, siguiendo las indicaciones de Ruíz (2002).

Los datos se recogieron por medio de la evaluación sumativa de los trabajos prácticos hechos en clase y los portafolios, según correspondiera a cada grupo. Se promediaron las 
notas para cada estudiante y en función de esos resultados, se aplicaron los análisis estadísticos pertinentes a cada grupo.

\section{Procedimiento}

\section{Las clases}

Los participantes cursaron igual programa de estudios y contenidos, con el mismo profesor y estrategias metodológicas. Estas consistieron en discusiones y explicaciones en clase en las que se trabajaron los contenidos y algunos ejercicios de ejemplificación. De igual forma, durante cada clase era obligatorio traer el artículo que los estudiantes estaban leyendo en cada unidad a fin de poder aclarar dudas ante el grupo en su totalidad. Adicionalmente, se realizaron ejercicios en el pizarrón, donde se aclararon también las interrogantes que se presentaron durante las clases.

Todos los estudiantes tenían una guía impresa suministrada por la docente, la cual al igual que el artículo científico también estaba disponible en la página Web de la profesora (atendiendo a las sugerencias que surgieron producto del estudio de Autor, 2008b). En esta guía se expuso tanto el cronograma de actividades (incluidos temas a investigar) como una serie de ejercicios por contenido programático a trabajar y evaluar. De la misma manera todos los participantes debían traer a la clase el artículo de investigación señalado con anticipación por la profesora para cada actividad. Éstos artículos fueron seleccionados en función de ciertos criterios específicos: eran provenientes de una fuente de alta credibilidad científica (en caso de ser electrónicos cumplían los criterios señalados por Espinoza, 2003 y Autor, 2008 ), complejidad o dificultad del vocabulario (determinada por la cantidad de léxico científico incluido), extensión del artículo y la familiaridad del tema para los estudiantes, especialmente considerando que eran cursantes del primer año y en su mayoría aún no tenían mucho conocimiento previo en las diferentes áreas especializadas relacionadas con su carrera. De esta forma se trabajó con artículos que discuten temas generales y que son tratados en otras asignaturas durante el primer año. Los artículos trabajados fueron sobre el incremento del riesgo de dolor orofacial por uso del cigarrillo y el chimó (Riley, Tomar y Gilbert, 2004) y sobre el uso de métodos dentales en la identificación de cadáveres calcinados en desastres masivos (Valenzuela, Martín, Marques, Expósito y Bohoyo, 2000). Los estudiantes 
manifestaron de forma espontánea que el vocabulario en los textos, así como la temática discutida en estos fueron fáciles de comprender.

Se contemplaron actividades de evaluación formativa para ambos grupos, previas a cada actividad a ser evaluada de forma sumativa. Además, la profesora disponía de un horario para asesorías a aquellos estudiantes que la requirieran para aclarar posibles dudas posteriores a la clase y previas a la actividad evaluada. Es importante aclarar que ningún grupo fue evaluado con exámenes de tipo tradicional. La única diferencia entre los ellos fueron las condiciones de evaluación: 1) portafolio y 2) trabajo práctico en aula, las cuales se especifican en detalle a continuación.

\section{La evaluación}

\section{Grupo evaluado con portafolio}

Los estudiantes tuvieron sus clases como se explicó con antelación. En su material de apoyo impreso y electrónico, se incluyó información acerca de los beneficios del uso del portafolio y las instrucciones de cómo realizar cada actividad, preparar y entregar el portafolio, así como la fecha de entrega del mismo. Esa información e instrucciones fueron dadas también de forma oral en clase tal y como lo recomiendan Villalobos (2002) y Autor (2008b) y se respondieron las preguntas de los estudiantes a fin de aclarar todas las dudas. Estas instrucciones se hicieron siguiendo las recomendaciones de Villalobos (2002) para un uso más productivo del portafolio. Además, se hicieron de forma oral y escrita y estuvieron disponibles en formato impreso y digital, siguiendo las recomendaciones de Autor (2008b).

Los lineamientos para hacer el portafolio se correspondieron con los señalados por Barragán (2005) previamente explicados en la sección de revisión de literatura en este artículo. El portafolio debía entregarse al final del período lectivo, lo cual daba a los estudiantes la oportunidad de realizar las evaluaciones de acuerdo a sus posibilidades de tiempo e inclusive de poder repasar un poco más antes de hacer la versión final a entregar.

Las características de las actividades a incluir portafolio eran las sugeridas por Barragán (2005). En este sentido, las tareas a incluir fueron:

1. Relacionadas con las experiencias de los estudiantes y por tanto relevantes para ellos.

2. Ajustadas a la duración de las clases y al curso como un todo. 
3. Perfectamente estructuradas y con una clara identificación de las competencias que se espera que desarrollen.

4. Buenas para facilitar aprendizaje individual y trabajo en equipo, $y$

5. Adecuadas para promover el pensamiento crítico y complejo.

\section{El grupo de evaluación en el aula}

Los estudiantes tuvieron clases y evaluación formativa similares a los del grupo de portafolio. Sin embargo, luego de la evaluación formativa, ellos tenían otra clase en la que realizaban la actividad evaluada en el aula. Las actividades eran las mismas del grupo de portafolio y también se correspondía con las características señaladas por Barragán (2005). Los estudiantes disponían de un lapso de dos horas para hacer las actividades y entregarlas en una hoja provista por la profesora para tal fin. Estas evaluaciones se hacían en presencia de la docente y podían hacer uso del material de apoyo que consideraran necesario (diccionario, traductores, listas de conectores, otros). No se permitía a los estudiantes consultar a sus compañeros y durante la actividad la profesora monitoreaba el grupo y aclaraba las dudas que los estudiantes tenían para que pudieran ejecutarla exitosamente.

\section{RESULTADOS}

El análisis de los datos se hizo utilizando el paquete estadístico SPSS 14.0 en español. Se consideraron algunos análisis descriptivos basados en información general de los participantes y se usó una prueba de comparación de medias para grupos independientes para establecer comparaciones en función de sus promedios y de esta forma observar el comportamiento de las variables implicadas. La información general acerca de los participantes se presenta en la Tabla 1.

Tabla 1. Información general acerca de los grupos.

\begin{tabular}{|c|c|c|c|}
\hline \multirow[t]{2}{*}{ Grupo } & $N$ & \multicolumn{2}{|r|}{$\mathrm{X}$} \\
\hline & & Edad & Nota \\
\hline Ejercicios prácticos en clase & 98 & $19,4(S=2,15)$ & $13,71(\mathrm{~S}=4,410)$ \\
\hline Portafolio & 98 & $19,2(S=1,87)$ & $14,80(\mathrm{~S}=3,152)$ \\
\hline
\end{tabular}

Revista EDUCARE, Volumen 14, Número 3, Septiembre-Diciembre 2010. ISSN: 2244-7296 
Como puede observarse en la Tabla 1, los dos grupos de participantes tienen características etáreas similares y la cantidad de participantes en cada grupo fue equitativa. En esta tabla se observa que los estudiantes en la condición de portafolio obtuvieron mejores calificaciones que los que trabajaron y entregaron las evaluaciones prácticas en clase.

Al observar las bases de datos, se apreció que la nota mínima y máxima para el grupo de actividades prácticas en clase fue de 06 y 19 respectivamente y para el grupo de portafolio fueron de 07 y 20, en la escala de 0-20 (Gráficos 1 y 2).

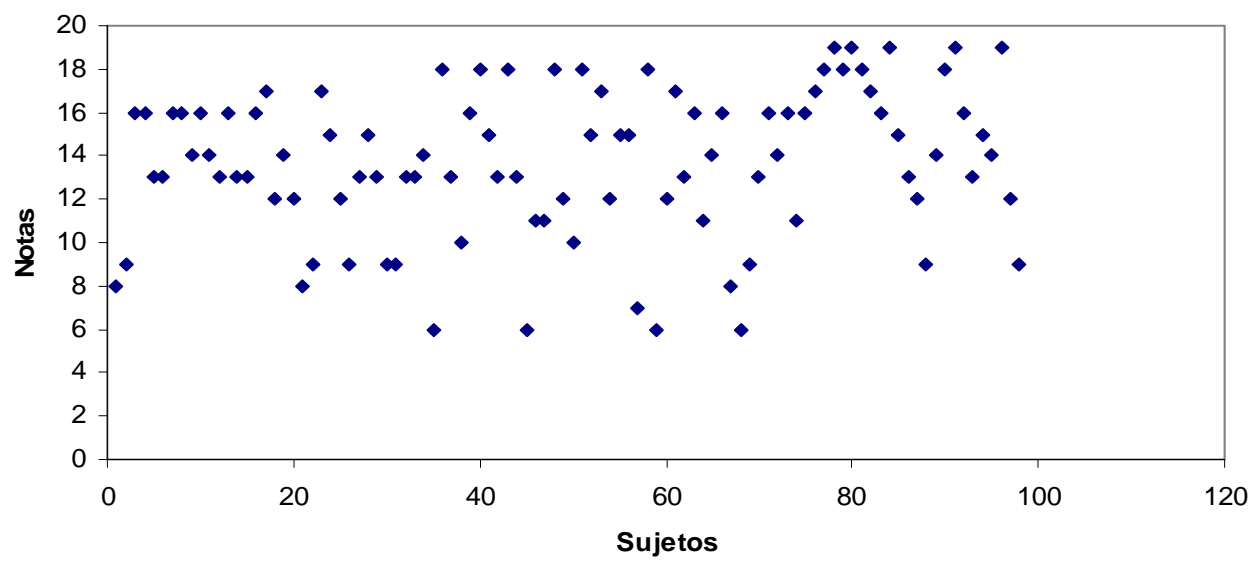

Gráfico 1. Distribución de las notas en el grupo de trabajo práctico en aula.

Como se puede observar, tanto en la nota mínima como en la máxima, también el grupo de portafolio superó al de actividades prácticas en clase.

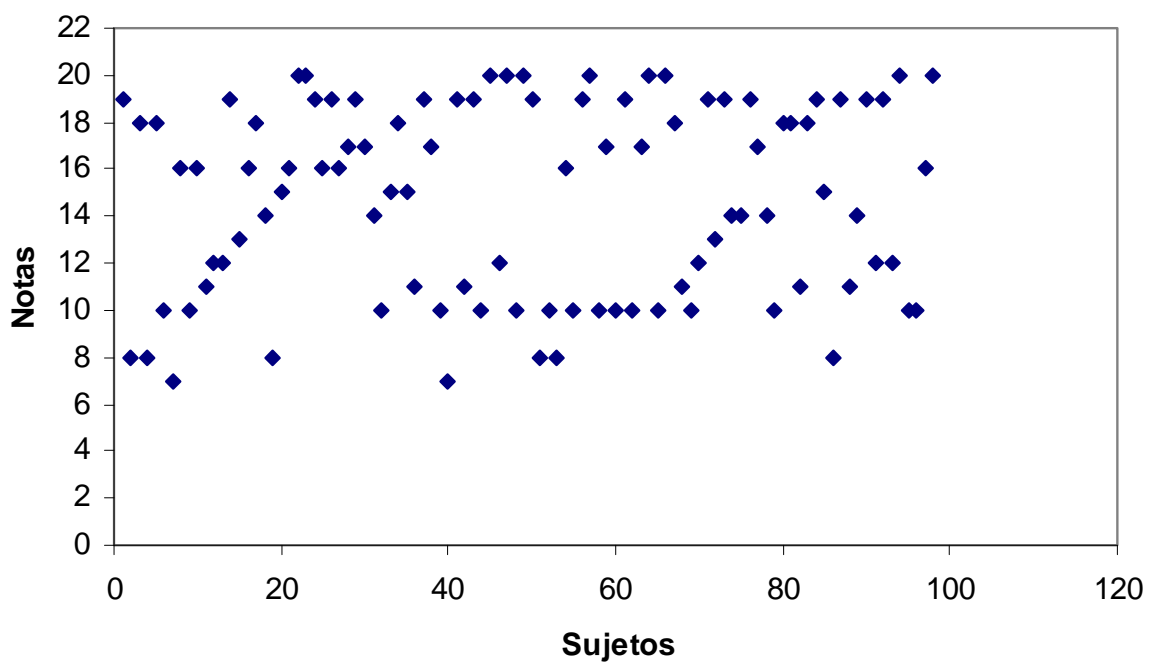

Gráfico 2. Distribución de las notas en el grupo de evaluación con portafolio. 
Para comparar apropiadamente las medias de ambos grupos y conocer cuál de las dos condiciones favorecían a los estudiantes, se hizo una prueba de comparación de medias, específicamente la t de Student. Los resultados de la misma se presentan en la Tabla 2.

Tabla 2. Resumen del resultado de la prueba $t$ de Student.

\begin{tabular}{cccc}
\hline$t$ & $n$ & $g l$ & $\mathrm{p}$ \\
\hline 1,6534 & 196 & 2 & 2,008 \\
\hline
\end{tabular}

La prueba se hizo a un intervalo de confianza de 95\%. Con el resultado se observa que hay diferencias significativas entre los dos grupos, en este caso a favor del grupo de portafolio.

\section{DISCUSIÓN Y CONCLUSIONES}

En la presente investigación, se compararon dos estrategias de evaluación que se ajustan a ambientes de enseñanza de IPE en contextos basados en premisas constructivistas. Los resultados comprueban la hipótesis planteada de que el uso del portafolio tendría una repercusión positiva sobre el rendimiento académico de los estudiantes, en comparación con las actividades de aula y dicha repercusión se ve reflejada en sus calificaciones.

La igualdad de los tamaños muestrales en ambas condiciones permitió hacer comparaciones de forma adecuada sin que posibles diferencias entre las muestras comprometieran estadísticamente los resultados. Igualmente, las semejanzas entre los grupos desde el punto de vista de sus edades permiten eliminar la posibilidad de que la edad sea una variable interviniente que modificara los resultados. El hecho de encontrar estas semejanzas entre los estudiantes de ambas cohortes (en cuanto a sus antecedentes educativos, proporción entre sexo femenino y masculino y edades, entre otros) guarda consistencia con los hallazgos de Autor (2007).

Autores como Delmastro (2005a y 2005b) han encontrado argumentos a favor del portafolio. Sin embargo, ni esta autora ni algún otro en la literatura revisada compararon esta estrategia con otras que se adecuen a un contexto de construcción de los aprendizajes en el 
contexto venezolano. En este sentido, es difícil establecer analogías y diferencias basándose en los resultados. Entre las pocas investigaciones con las que se puede hacer una comparación aproximada con el presente estudio se encuentra la de Makarchuck (2003) quien comparó el portafolio con otras formas de evaluación. Los presentes resultados difieren del de Makarchuck, ya que en él no encontró diferencias significativas en relación con otras formas de evaluación.

Sin embargo, el autor citado señaló que sus resultados presentaban problemas al momento de generalizarlos dado el pequeño tamaño de la muestra. A diferencia del trabajo de Makarchuck (2003), en la presente investigación es más factible generalizar, ya que el tamaño muestral fue notablemente mayor. Adicionalmente, las diferencias entre los resultados de ambas investigaciones podrían obedecer también tanto a diferencias culturales (dados los contextos geográficos en los que se hicieron) como a ciertas diferencias metodológicas; por lo que sería interesante seguir investigando al respecto, tomando en cuenta los detalles que pudieran haber comprometido sus resultados (por ejemplo, su diseño y tamaño muestral) y que pudieran hacerle poco comparables con el presente estudio.

Sobre la base de los hallazgos de la presente investigación es posible señalar que el uso de portafolio favorece a los estudiantes de IPE, a la vez que representa una alternativa de evaluación. Por consiguiente se recomienda su uso en contextos similares al de la presente investigación, pese a que algunos han afirmado que esta estrategia demanda mucho esfuerzo y tiempo por parte de los docentes que administran los cursos (Vera y Canalejas, 2007). Ante ese esfuerzo necesario para el uso del portafolio en el aula Song y August (2002) recomiendan que haya alguien que ayude al docente en la evaluación de los portafolios. En algunos contextos es difícil poder ejecutar esa sugerencia, entre otras cosas, por las carencias presupuestarias que enfrentan las universidades.

No obstante, Autor (2008b) presenta una alternativa más viable en nuestro contexto que sería incluir en el portafolio sólo unas pocas actividades. Es decir, que el portafolio sea una de las estrategias de evaluación en el curso pero no la única. De esta manera se podrán incluir en éste aquellas actividades que se ajusten a la naturaleza de la estrategia y buscar otras formas de evaluación que también se adecuen a ambientes constructivistas para el resto de los contenidos programáticos. 
Es posible que los hallazgos del presente estudio se hayan visto influenciados por la falta de compromiso en los estudiantes y por fallas al momento de seguir instrucciones tal y como se observó en los resultados de Delmastro (2005ª). En este sentido, se recomienda al igual que se hace en el estudio de Autor (2008b), motivar a los estudiantes en cuanto al su uso y fomentar en ellos la responsabilidad que implica realizar los portafolios de acuerdo a las instrucciones dadas. De esta forma serán ellos mismos quienes administren el tiempo y decidan el momento en que completan las actividades a incluir.

Las notas mínima y máxima en la condición de portafolio fueron superiores a las del grupo de actividades prácticas en aula, pese a que los estudiantes estaban más familiarizados con esta última estrategia de evaluación. Es posible pensar, sobre la base de esos resultados, que el hecho de que el portafolio permita al estudiante trabajar con comodidad e ir incorporando los trabajos cuando se sienta listo, pudiera generar que el producto final sea de mayor calidad. Sin embargo, debería investigarse en mayor detalle las variables involucradas en dicha hipótesis.

Adicionalmente, se recomienda tomar en cuenta las sugerencias de Autor (2008b) y Delmastro (2005ª $)$ en cuanto a seguir trabajando en función de una mayor autonomía y compromiso en los estudiantes para la preparación y presentación del portafolio. Paralelamente, sería de interés indagar acerca de los factores que afectan el nivel de compromiso en el su uso, lo cual no fue considerado en esta investigación ni lo ha sido en ninguno de los antecedentes hallados en la literatura consultada.

Se considera oportuno seguir incorporando el uso del portafolio como alternativa de evaluación en contextos constructivistas, ya que de esta manera los estudiantes tienen mayor oportunidad de gerenciar su propio proceso de aprendizaje, se ven beneficiados por la evaluación formativa y pueden trabajar en función de su disponibilidad de tiempo. Los resultados en este estudio han ilustrado un impacto positivo del portafolio sobre las calificaciones, pese a que era una estrategia con las que los estudiantes estaban poco familiarizados. Si se logra que los alumnos usen de forma adecuada el portafolio, no sólo se verían beneficiados en función de las calificaciones, sino que adicionalmente fortalecerían su independencia y autonomía en el proceso de aprendizaje, tal y como se espera que suceda en ambientes constructivistas. En este contexto, es importante que los docentes sigan trabajando 
para ayudar a los estudiantes a darse cuenta de que una manera de aprovechar efectivamente el uso del portafolio es respetando las instrucciones para su llenado y entrega las cuales deben ser dadas a través de diversos medios, incluidas las tecnologías de la información y comunicación.

Por último, se cree importante que el uso del portafolio no se limite a la universidad sino incorporarlo en contextos educativos previos, pues a los estudiantes universitarios se les dificulta un poco porque en muchos casos esa es su primera experiencia con esta estrategia. Es decir, si se comienza a trabajar con portafolios (aún de grupo) en los ámbitos educativos previos al universitario (así como se usan las tareas en el aula) será más sencillo para ellos adecuarse a la manera eficiente de usarlo. Finalmente, se concluye también que el portafolio es una estrategia de la que mucho falta aún por investigar y decir; y dadas las implicaciones pedagógicas de su uso es necesario continuar investigando al respecto en diferentes contextos en el campo de ILE.

\section{REFERENCIAS}

Akirov, A. (1997) Algunas reflexiones sobre el uso del portafolio como alternativa confiable en la evaluación de los aprendizajes. Entre Lenguas. 1 (3), 39-41.

Barragán, R. (2005) El Portafolio, metodología de evaluación y aprendizaje de cara al nuevo Espacio Europeo de Educación Superior. Una experiencia práctica en la Universidad de Sevilla. Revista Latinoamericana de Tecnología Educativa. 4 (1), 121-139.

Delmastro, A. (2005a) El portafolio como estrategia de evaluación en la enseñanza de lenguas extranjeras: fundamentos teóricos y orientaciones procedimentales. Lingua Americana. $16,43-68$.

Delmastro, A. (2005b) El uso del portafolio en la enseñanza de lenguas extranjeras: perspectiva del docente. Investigación y postgrado. 20 (2), 187-211.

Delmastro, A. y Salazar, L. (2004) Tendencias en la enseñanza de lenguas extranjeras: hacia un eclecticismo constructivista. Sinergies Venezuela, 3: 19-37.

Espinoza, N. (2003) Criterios para la selección de información científica odontológica en la World Wide Web. Acta odontológica. 41 (3), 251-257.

Floréz, R. (1999). Evaluación pedagógica y cognición. Bogotá: McGraw Hill.

Kavaliauskiene, G., Kaminskiene, L y Anusiene, L. (2007) Reflective practice: assessment of assignments in English for Specific Purposes. Ibérica. 14, 149-166. 
Lau, S. (2006) The implementation of portfolio assessment in an ESL/EFL Classroom. En H. Nguyen and J. Kirshenmann (Eds.), TESL Working Paper Series. (pp. 25-30). Hawaii: Hawaii Pacific University.

Lunar, L. (2007) El Portafolio: Estrategia Para Evaluar La Producción Escrita En Inglés Por Parte De Estudiantes Universitarios. NÚCLEO. 24, 63 - 96.

León, O. y Montero, I. (1997). Diseño de investigaciones. Introducción a la lógica de la investigación en psicología y educación. 2da Ed. Madrid: Mc Graw Hill.

Makarchuk, D. (2003) Portfolio use and EFL Learning and assessment in South Korea. The Korean TESOL Journal. 6 (1), 39-57.

Nunes, A. (2004) Portfolios in the EFL classroom: disclosing an informed practice. ELT Journal. 58 (4), 327- 335.

Öztürk, H. y Çeçen, S. (2007) The effects of portfolio Keeping on writing anxiety of EFL students. Journal of Language and Linguistic Studies. 3 (2), 218-236.

Peña, J.; Ball, M. y Delhi, F. (2005) Una aproximación teórica al uso del portafolio en la enseñanza, el aprendizaje y la evaluación. Educere. 9 (31), 599-607.

Autor, B. (2007) Estrategias de lectura usadas por los estudiantes de nuevo ingreso de la Facultad de Odontología de la Universidad de Los Andes, Venezuela. Kaleidoscopio. 4 (8), 153-161.

Autor, B. (2008a) Búsqueda y selección de textos en la Internet para investigación científica. Saber. 20 (1), 133-140.

Autor, B. (2008b) Assessing learning through portfolio use by university students. The experience of an ESP class in Venezuela. Ponencia presentada en el ICERI 2008. Madrid, España.

Reeves, T. y Okey, J. (1996) Alternative assessment for constructivist learning environments. En B. Wilson (Ed.) Constructivist Learning Environments. Case Studies in instructional design. (pp. 191-202). New Jersey. Educational Techonology Publications. Englewood Cliffs.

Riley, J., Tomar, S. y Gilbert, G. (2004) Smoking and smokeless tobacco: increased risk for oral pain. The Journal of Pain. 5, 218-225.

Ruíz, C. (2002). Instrumentos de investigación en educación. Procedimientos para su diseño y validación. 2da Ed. Caracas: CIDEG.

Song, B. y August, B. (2002) Using portfolios to assess the writing of ESL students: a powerful alternative? Journal of Second Language Writing. 11 (1), 49-72.

Valenzuela, A., Martín, S., Marques, T., Expósito, N. y Bohoyo, J. (2000) The application of dental methods of identification to human burn victims in a mass disaster. International Journal of Legal Medicine. 11, 236-239.

Vera, M. y Canalejas, M. (2007) El portafolio como recurso de aprendizaje e instrumento de evaluación de estudiantes repetidores de enfermería. Educación Médica. 10 (2), 114120. 
Villalobos, J. (2002) Portafolios y reflexión: Instrumentos de evaluación en una clase de escritura. Educere. 5(16), 390-396. 